\title{
Theoretical Organization of Motivations to Attend First Aid Education: Scoping Review
}

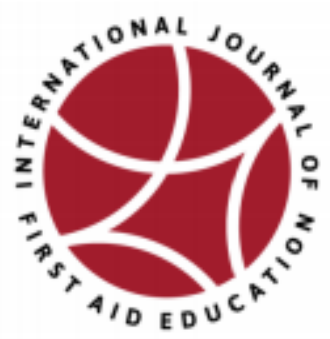

Jeffrey L. Pellegrino, Ph.D./MPH, Aultman College of Nursing \& Health Sciences

Nicholas Asselin, D.O., Brown University

First Aid Education serves the public's health to prepare for and respond to acute illness and injuries and to return people to health. Understanding motivations for people to participate in education opportunities would help organizations contextualize learners' expectations and be used to increase the percentage of the public trained to respond. Using PRISMA guidelines, we identified existing literature on individual and/or population motivations to participate first aid education and motivational factors to seek education, information, and training to prepare to respond to emergencies from observational studies.

The results however did not lend to a cohesive body in which metanalysis could be performed. We present a scoping review of literature mapped to current social and psychological theories for context and use in developing or promoting first aid education. We identified three smaller experimental studies and no large experimental or population-based studies. Two observational or retrospective studies and eight descriptive studies rounded out the literature that included motivation to attend. Motivations to participate in first aid education are not well described in the literature in terms of magnitude or generalizability. Those identified do fit into behavioral theories, as applied in the Social-Ecological and Transtheoretical models for the benefit individual educators and training organizations.

Linking theory to motivations ideally helps develop future research in the field from psychological and sociological perspectives. Practically, there is opportunity for better research connection between education organizations who can "require" training for occupational or educational purposes to increase awareness and opportunity for first aid education. Public health educators can also link first aid education to other public health topics or vice versa to weave in community motivations for competencies to build resilience in personal or public emergencies.

The "Chain of Survival Behaviors" (International Federation of Red Cross Red Crescent Societies, 2016) identifies the domains of first aid education (inclusive of Cardiopulmonary Resuscitation (CPR) education). The linear nature of preparing for, responding to, and recovering from acute illness or injury provides opportunity for micro and comprehensive first aid education (see Figure $1)$.

As first aid educators, we seek to empower individuals and communities to develop and be willing to enact helping behaviors and care for an acute illness or injury (Pellegrino et al., 2017).
How public health educators and learners come together is a matter of logistics and circumstance to a certain extent, but the individual must ultimately be motivated to attend first aid education. As first aid education matures from content focused to learner focused, learner motivations and behaviors become more important to the success of our work in creating personal and community resiliency in health emergencies.

Motives to preserve life, alleviate suffering, prevent further illness or injury, and promote recovery, which are goals of first aid (Singletary et 


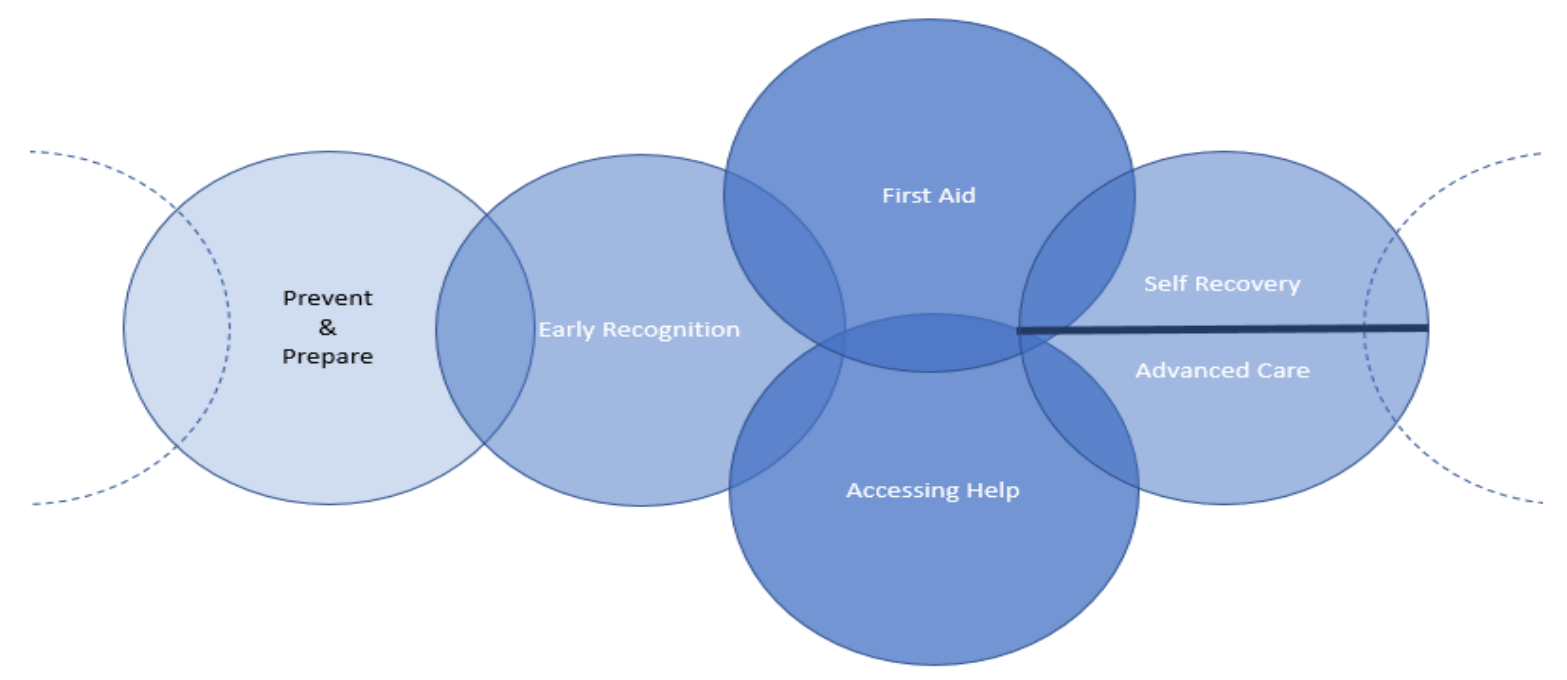

Figure 1: Chain of Survival Behaviors

al., 2015: S575) might be seen at the individual, organizational, or cultural levels but may not sufficiently describe motives for deciding to attend an educational program/course.

To improve the dissemination of first aid education, a better understanding of the motives of learners to seek training is imperative for population saturation of knowledge, skills, and resources to respond to emergencies. Practically this would contribute to financial solvency of training organizations.

To date, no body of evidence exists to describe motivations experimentally to determine which influence behaviors to attend first aid education programs or other learning outcomes. This scoping review allows others to look more broadly at known motivations and their strength along with effectiveness of specific motivations for individuals to attend first aid education. Our goal is to synthesize the existing research evidence and identify appropriate behavioral models for which they may be mapped for use by others in research. At the community/ lay responder level, our guiding questions included:
- What motivations exist for individuals/populations who participated in "first aid" education?

- What factors motivated learners to seek education, information, and training to prepare to respond to emergencies from observational studies?

\section{Methods}

The American Red Cross Scientific Advisory Council (SAC) approved this inquiry in June 2018 and the final review in January 2019. Our search strategy incorporated best practices established in Preferred Reporting Items for Systematic reviews (Moher et al., 2009) and Meta-Analyses extension for Scoping Reviews (PRISMA-ScR) Checklist (Tricco et al., 2018), which follow the SAC peer review protocol(Science Behind Our Programs | Science Advisory Council | Red Cross, n.d.) to openly communicate the process for gathering literature for review. Our review team only considered observational or experimental evidence in the form of peer reviewed manuscripts in scholarly journals. To establish a scoping review of the literature we did not establish a time limiter, however because the initial search was 


\section{Scientific Advisory} Council

The American Red Cross Scientific Advisory Council is a 50+member panel of nationally-recognized medical, scientific, and academic experts dedicated to ensuring that the Red Cross is at the vanguard of best-science and best-practice.

With members from a broad range of professional specialties, the Council has an important advantage: a broad multidisciplinary expertise for evaluating new emergency response methods and techniques. And with on-the-ground experience, its members also bring the know-how for real-world emergencies. Whether it's the best way to administer CPR to an unconscious co-worker or preparing your family and home for a hurricane, the Council is keeping you current.

This review was approved by the Educational Sub-council and full Council in January 2019.*

*The Editor-in-Chief, and Senior Editors of the IJFAE participate on the Scientific Advisory Council.

conducted through electronic databases there was some limitation to the available sources; with the

earliest work identified in 1965. Self-limiting the search also occurred regarding language, as English or English translations of titles and abstracts were needed to be included in the full paper review.

\section{Database Search Strategy}

We chose the EBSCO Discovery Service of Kent State University which searched 99 data bases, utilizing key words of "first aid" AND "motivation" because first aid education may be indexed in a variety of disciplines beyond health, including business, sports, and education. The most recent search, on 24 January 2019, yielded 365 results with duplicates removed. We also searched Pubmed database directly to verify accuracy of initial search string. From the results, we hand searched studies citied within other studies, yielding 50 additional manuscripts (see Figure 2).

\section{Screening Process}

The primary authors independently screened title and abstracts of the search results for initial inclusion. We included a study if it identified a learner's motivation(s) to participate in first aid education at the lay or community level. Health care professionals or health care students were excluded due to career motivations not being independent of other motivations. A moderate degree of agreement was achieved by co-authors (84.39\%, Cohen's k: 0.44). Ninety-five were excluded because either the title or abstract was non-English and inclusion criteria could not be established. The co-authors then discussed differences and came to an agreement on inclusion or exclusion for full paper review without the need of a third party. Titles or abstracts that were potentially contributing were left in for the full paper examination. Original data were not collected, nor expected in this review.

\section{Summary of Included Studies}

We included thirteen published studies for final analysis and expert review by the primary authors, confirmatory approval by the Education Subcouncil, and peer review by the full SAC (see Table 1). Countries represented in the analysis: Australia (3), Austria (1), Belgium (1), Canada (1), China (1); 


\section{Identification \\ Records identified through database searching $(n=365)$ \\ Additional records identified through other sources $(n=50)$}

\section{Screening}

Records after Duplicates Removed ( $n=396)$

Records Screened by title \& abstract $(n=396)$

Records Excluded $(n=302)$

95 non-English

12 for document type (appendix, index)

\section{Eligibility}

Full-text articles assessed for eligibility ( $n=60)$

Full-text articles excluded, with reasons ( $n=46$ )

2 were review articles for which original articles were used

4 were English only abstract for which detail wasn't available

rest were not on motivation to attend a course, but different motivations (to act or for acting)

\section{Included}

Studies included in qualitative synthesis $(n=14)^{*}$

Studies included in quantitative synthesis $(n=0)$

* One study excluded during the analysis due to indirectness

Figure 2: PRISMA Reporting Schema

Note: PRISMA guidelines and reporting are used within internal documents of the American Red Cross Scientific Review protocol and report on during the peer review process to experts in public health education and clinical practice.

France (1), Scotland (1), Taiwan (1), and the United States (3) There were no studies that experimentally explored motivations to participate in first aid education. Eight of the studies were survey-based with motivation variables chosen from a list of options but not theoretically established as an a priori outcome or explained in discussion.

Pearn, et al. (1980) explored the notion of time (length) of a first aid course and costs as either motivators or barriers to attendance of people who recently purchased a swimming pool, in Australia. Only $9 \%$ of the 700 invited participated overall in the education offered. Cost appeared to be a factor in which courses participants chose; showing that the perceived value of first aid courses did not match high costs. A surprise to the researchers, similar percentages of people signed up for 1-3-hour courses as a course of eight 3-hour sessions over 4 weeks, with 100\% completion. Reported characteristics of participants in the education over $75 \%$ included: having children and never having experienced the need for CPR. For an AED (automated external defibrillator) program in sports clubs, cost also came up as a motivator for participation (Fortington et al., 2017).

Greenberg et al. (2012) compared a group receiving a prescription for a CPR Anytime (C) selflearning kit, consisting of a CPR manikin and a 22- 
minute DVD and another group prescribed a CPR class. Across both investigational arms, 15\% $(12 / 77)$ followed prescription advice to purchase the CPR kit and $0 \%(0 / 79)$ took a CPR class. Researchers did not parse out motivation, but for at-risk populations who received educational prescriptions, the at home/self-training kit yielded more learners than those who would need to logistically go to a class after an illness demonstrating some efficacy for self-learning.

Charlier \& De Fraine (2013) differentiated a group's motivation to learn first aid after experiencing a game that raised the subject, compared to a control group. Results favored the game introduction and motivation to attend future first aid courses $(86 \%$ v. $82 \%)$.

To learn if having an at-risk family member for cardiac arrest influences motivation to learn first aid, Kliegel, et al. (2000) asked a combined sample of 87 to participate in a 1 -session CPR course. Medical students offered courses either in a training room environment or in-home. Only two of the target group and zero of the control group had taken CPR in the last year out of the approximately $50 \%$ who had taken CPR previously. Twice as many target group participants chose the at-home venue for training, compared to the control group.

From survey data in a mixed study of healthcare workers asked about providing care in emergencies outside of work, Arbon, Hayes \& Woodman (2011) determined that motivation to attend a course came from it being a workplace request or requirement (44.3\%) and assisting family and friends (31.1\%). Zacharatos, Barling \& Iverson (2005) in a meta-analysis of high performance work systems and safety identified trust in management and perceived climate for safety as mediating factors of safety performance, which included first aid knowledge, motivation, and compliance. Huang, et al. (2016) created a logistic regression model for investigating the characteristics of student and public respondents in Wuhan, China $(n=1407)$ that influenced their willingness to learn CPR. It revealed that family members having a cardiovascular disease (yes vs. no, $\mathrm{OR}=2.67$ ), opinion of development of local medical emergency system (perfect vs. poor, OR $=3.15)$, having ever heard of $\mathrm{CPR}(\mathrm{OR}=2.43)$ were independently associated with willingness to attend CPR education. Characteristics of gender, educational level, or CPR knowledge were also in the model but don't represent initial motivational characteristics.

Asked qualitatively about their motivations to participate as Community First Responders in rural Scotland, they expressed enthusiasm for contributing to their community (Roberts et al., 2014). Statements of bridging the gap between health professionals and the community and providing support while awaiting ambulance arrival were given. Some had first aid knowledge:

I became a first responder just to give something back to the community and also because I do basic life support training at work, to other people and I just thought it was a good way of maintaining it for myself and actually using it. (Focus Group 2, p. 5).

Experience of types of previous emergency situations influenced some:

My dad took a heart attack and I had no idea what to do .....I want to try and help somebody because you've got no chance up here if you're, say half an hour away from the hospital.....that's the thing that pushed me into doing it. (Focus Group 6, p. 5).

Well I joined because being a fire fighter you're helping the community, so it's just to, further help for the community and, you never know when you need the service yourself. (Focus Group 5, p. 5). 
Socially, Fortington et al. (2017) also identified a duty of care as a key driving factor, with recognition of AEDs as an asset to communities broadly, not just the participants' immediate sports setting. Macro opportunity (free) training awareness led participation by organization. A legal or moral duty of care held by their clubs/facilities emerged as a strong motivator in the decision to apply to the AED program. This duty was perceived to extend to both club members and the public attending club events or passing by the location. Assistance with the cost of AED acquisition also emerged as a significant motivator contributing to decision to apply to the AED program.

Other survey articles also identified main motivations to participate in education as wanting to have a useful skill and being prepared for emergencies (Bouland et al., 2017; Yang et al., 1998). Family members of patients with heart disease, when surveyed about their attitudes toward CPR reported CPR training because of a school or job requirement $(59 \%, n=49)$, while only $8 \%(n=4)$ stated the primary motivation was living with someone at risk for out-of-hospitalcardiac-arrest (OHCA) (Platz et al., 2000). Similarly, Cariou \& Pelaccia (2017) retrospectively interviewed family members of at-risk individuals finding that $65 \%$ received training due to professional and military duties, while only 3.5\% because of living with an at-risk person.

\section{Discussion}

The quality and disparate nature of the studies in terms of design and outcomes did not lead to any ability to discern with confidence population applicable motivational factors to attend first aid education. Motivation in general was not the main outcome of interest in the studies with interventions and reported without insights from theory or importance to other outcomes. Characteristics of learners more likely to participate or willing to learn were described in a few studies (Greenberg et al., 2012; Huang et al.,
2016; Kliegel et al., 2000). Being female or having a higher educational level might be characteristics of learners in general. Characteristics of having a family member with cardiovascular disease or knowing that CPR as a technique exists may be more specific toward first aid education. Finally, an existing knowledge base or experience with content or Emergency Medical Systems (EMS) system supports further learning.

This review focused on motivations that instilled action for some degree of first aid education from a public setting (e.g., non-healthcare professions) and to help discern the outcomes observed in these studies we have chosen to thematically arrange them in context of behavioral theories and models. At face value the motivations can be broken into three domains: Extrinsic, Opportunistic, and Intrinsic (see Figure 3). This simple breakdown provides first aid educators insights into which motivations might be generated through policy (i.e., requirements); those that be useful in targeted recruitment; and those that might be of a general environmental nature.

Extrinsically, legal and compulsory requirements were a strong motivation for participation. Specifically, as a job or school requirement (Arbon, 2011; Cariou, 2017; Platz, 2000). Weaker evidence for duty to care in sports (Fortington, 2017) and for regaining an expired certification exists (Bouland, 2017). From a moral/ethical perspective motives to help others, including a prescription or advice from others were weak in supporting evidence (Cariou and Pelaccia, 2017; Greenberg et al., 2012; Platz et al., 2000). Environmentally, there was neutral evidence to suggest that high performance systems/safety culture motivate participation in first aid education (Zacharatos, 2005). Weaker evidence identified distance from help (Fortington, 2017), payment from work to attend (Arbon, 2011), and participation in sporting activity (Arbon, 2011) as potential motivations to learn. 
Opportunistic motivations exist as there was not an identified contemplation prior to the opportunity to participate. Cost and length of course (Pearn, 1980) were of a neutral value for participation, and weak evidence exists for training of peers/family with a free kit (Ikeda, 2016). Ikeda also found indirectly a "Pay it forward" ethical stance for people taking a course opportunistically. Convenience of taking a course led others, in a neutral perspective, to participate (Platz, 2000).

Intrinsic Motivation for altruistically helping family and friends (Arbon, 2011) and to be prepared just in case (Bouland, 2017) had strong associations for motivating people to attend and learn. Strong but indirect evaluation supported this in survey studies (Cariou, 2017; Kanstad, 2011) and weak and indirect evaluation exists too (Ikeda, 2016, Platz 2000). Contribution to the community as a motivation was identified from a weak study (Roberts, 2014). Environmentally, weak evidence exists for motivations to help a vulnerable family member (Bouland, 2017; Carious, 2017; Kliegel, 2000; Platz, 2000), and indirectly helping someone with cardiac disease (Huang, 2016; Ikeda, 2016; Kanstad, 2011) exists. Although strong, indirect evidence suggests that one's opinion of EMS/knowing what CPR is motivates participation (Huang, 2016). Weak evidence does suggest that one can be selfmotivated to attend (Arbon, 2011). Strong evidence exists that making first aid a requirement motivates future participation, but it is not described as to why this characteristic exists. There may be motivations to continue to be prepared and a value for practice/ validation through maintaining a first aid certificate or simply a continuation of a requirement for work/ education.

The Transtheoretical Model (TTM) (Prochaska and DiClemente, 1983) proposes different stages that individuals reside in term of establishing a regular habit of behavior, in this case maintaining first aid competencies. Motivations could be the reason for moving from one state to the next (see Table 2). The benefit from seeing motives for first aid educators is to understand supportive and weak motives, which is not defined in the literature yet, that can be utilized to support individuals from a known stage to another. Teachers, counselors, sales representatives, supervisors can demonstrate mindfulness in recognizing common motivations and match them with opportunities for moving toward maintenance outcomes.

The Social-Ecological model (SEM) (Bronfenbrenner, 1979) (see Figure 4) provides

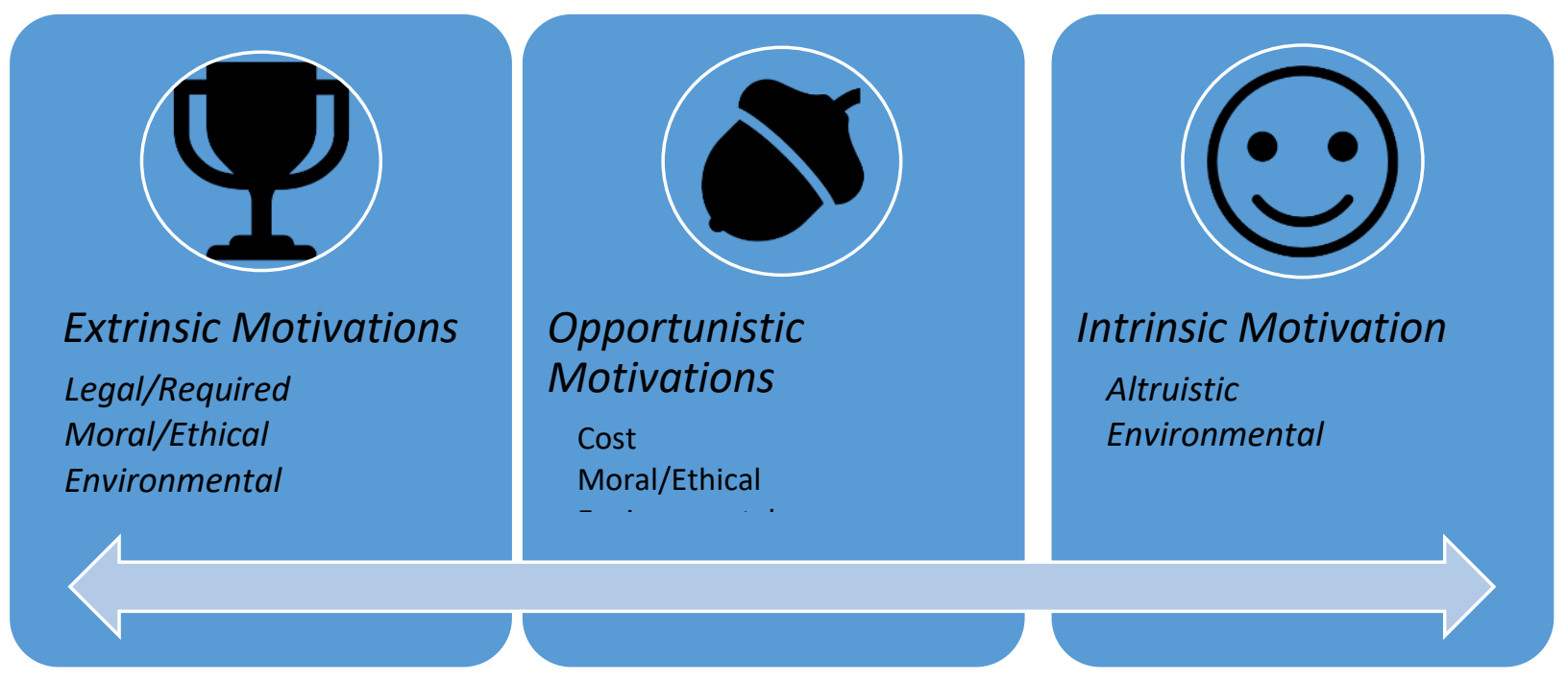

Figure 3: General categories of motivations to participate in first aid education 
curriculum designers and policy makers for first aid a spatial understanding of where motives can be generated and influenced socially to increase first aid education behaviors of the individual. Working in concentric circles out, starting with the individual/population altruistic motivations of helping family/friends and wanting to be prepared were found to be present in the stronger studies (Arbon et al., 2011; Bouland et al., 2017). Within the microsystem, those most proximal to the individual, requirements for job or school motivated attendance in stronger studies (Arbon et al., 2011; Cariou and Pelaccia, 2017; Platz et al., 2000). Prescriptions from physicians, payment from work, and participating in sporting activities did not have strong evidence as motivating factors to attend (Arbon et al., 2011; Fortington et al., 2017; Greenberg et al., 2012; Platz et al., 2000).

The exosystem consists of organizations that create the lived environment of the individual, but not always directly involved. The presence of the Red Cross in a community with trainers is an example of this. How a training organization markets to individual or microsystem and then makes themselves accessible can use motivations to their advantage, for example in the AED program for sports clubs provided opportunity to the individual to participate (Fortington et al., 2017).

The macrosystem, which includes culture, mores, laws, etc. surround all other systems. Exosystem organizations have opportunities to influence policy, create conversations, and move public opinion through social media, policy development, or politically in the macrosystem as advocates. First aid education as a job or school requirement motivates people to attend (Arbon et al., 2011; Cariou and Pelaccia, 2017; Platz et al., 2000 ) and is an active tool of organizations working to influence policy (International Federation of Red Cross Red Crescent Societies, 2015).

Bronfenbrenner (1994) calls out the mesosystem as means for assessing competing or cooperative forces on a person's behaviors. The more cooperative the easier it is for a person to make or maintain behaviors. In this case, the opportunity for first aid educators is to help make things align between organizations and individual. For example, sporting clubs being able to have access to AED, leads to training, and training being available at no cost is an alignment that connects and organizes larger cultural motivations like duty to care to opportunistic motivations around cost

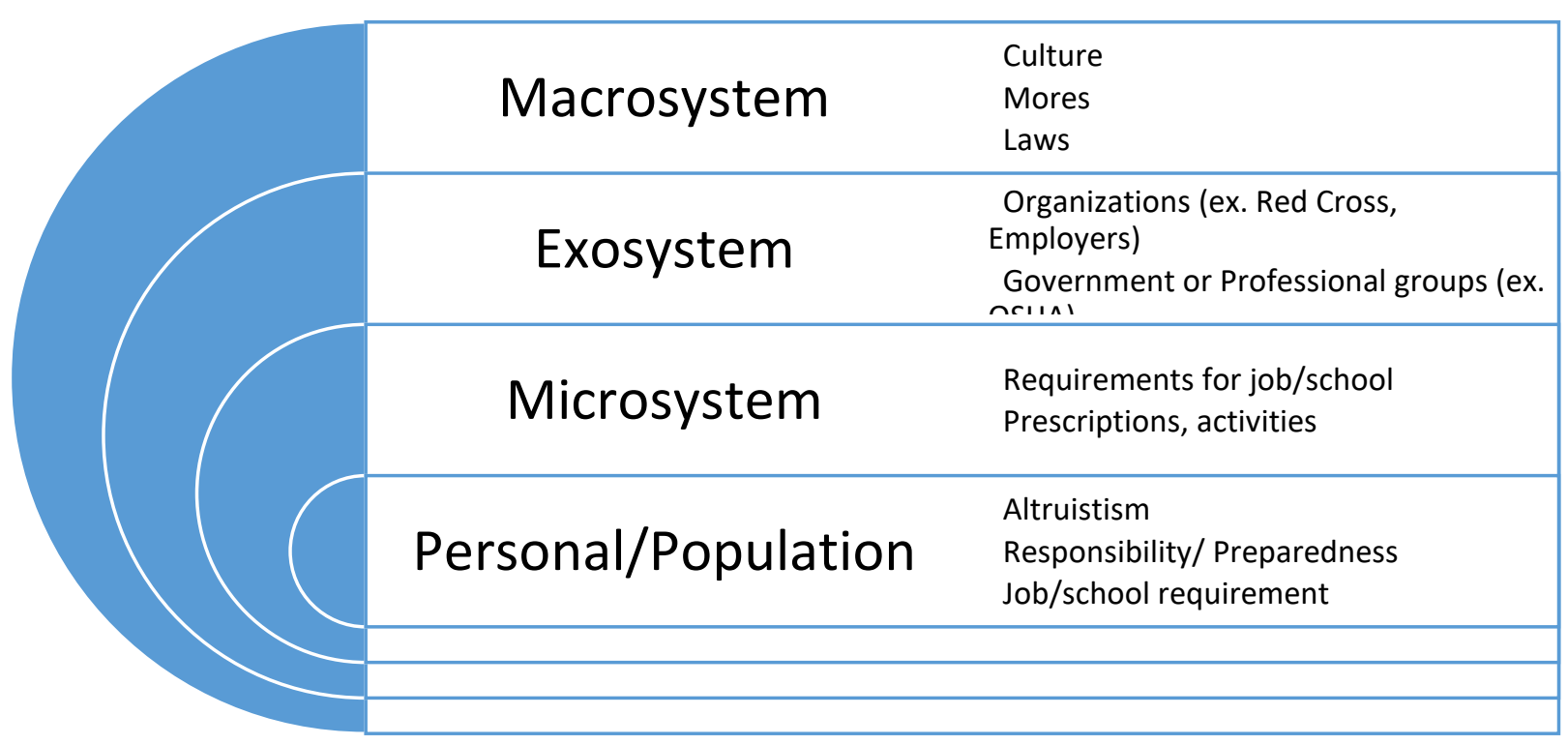

Figure 4: Social-ecological relationships for motivations to participate in first aid education. 
and access of training (Fortington et al., 2017). High performance work systems (Zacharatos et al., 2005) provides another example of how the mesosystem can influence safety behaviors, in which first aid education has a role.

Missing from this analysis is the chronosystem that illustrates that people/populations over time have different needs and motivations for first aid education. Games that encouraged exploration of first aid (Charlier and De Fraine, 2010) could be in a microsystem at school but could also be limited to adolescents in developing a motivation to learn more. Similarly, chances of living with someone who is at risk for cardiac arrest increase with age leaving more motivation, but fewer physical abilities.

Overall, there is limited and low-level evidence of poor quality to answer the specific question of what motivates people to participate in first aid education in any monolithic terms. There was also a lack of studies of motivations to participate in non-facilitated educational opportunities (e.g., airport compression CPR practice stations). Most studies asked the question without any behavioral model or theory behind motivations to participate or demonstrated validity in their measures of motivations. This scoping review had limitations in terms of accessing non-English manuscripts for inclusion. This review, although inclusive of international contributions, has been presented from a western behavioral perspective and not inclusive of other large cultural perspectives in the world. There also maybe other appropriate behavioral or psychological models for which people can understand, measure, and utilize for motivating individuals and populations to participate in first aid education.

\section{Conclusion}

From a scoping review of motivations to attend first aid education, as reported by participants, there has been little attention to learning the role of motivation respective to increasing personal participation or involving populations at theoretical or practical levels. Strong evidence exists that making first aid a requirement motivates participation in education, along with meeting altruistic needs of some, to be prepared for emergencies, especially family and friends. After initial exposure to first aid education there is a gateway opportunity to motivate people to continue to learn first aid in deeper contexts and/or regularly to maintain competencies. This organization of motivations should challenge education organizations to not only recruit more efficiently and effectively but foster relationships with learners and more fully prepare them to respond appropriately through the other domains of the Chain of Survival Behaviors.

Future work in looking at the strength of motivation(s), not just a presence of one or another, would support first aid education's development toward learner centered experiences (Pellegrino et al., 2017) both psychologically and socially. Training organizations, like the American Red Cross, can better serve individuals and populations by tailoring experiences with evidence-based content based on motivation(s) to attend. The value of mapping current motivations to established behavioral models with theoretical foundations allows for future research to better use or define gaps that could prevent or encourage people's participation. Motivations to participate can also inform course learning outcomes (e.g., willingness, confidence, attitude) giving curriculum designers and educators context for which to engage and then assess success of the education. 
Review Article

\section{References}

Arbon P, Hayes J and Woodman R (2011) First aid and harm minimization for victims of road trauma: a population study. Prehospital and Disaster Medicine 26(4)276-282. DOI:

10.1017/S1049023X11006522; 10.1017/S1049023X11006522.

Bouland AJ, Halliday MH, Comer AC, et al. (2017) Evaluating Barriers to Bystander CPR among Laypersons before and after Compression-only CPR Training. Prehospital Emergency Care 21(5). DOI: $10.1080 / 10903127.2017 .1308605$.

Bronfenbrenner U (1979) The Ecology of Human Development : Experiments by Nature and Design. Cambridge, Mass: Harvard University Press.

Bronfenbrenner U (1994) Ecological Models of Human Development. In: Gauvain M and Cole M (eds) Readings on the Development of Children. NY: Freeman, pp. 37-43.

Cariou G and Pelaccia T (2017) Are they trained? Prevalence, motivations and barriers to CPR training among cohabitants of patients with a coronary disease. Internal and Emergency Medicine VO - 12 (6). Springer: 845. DOI: 10.1007/s11739-016-1493-8.

Charlier N and De Fraine B (2010) Games based learning as a vehicle to teach new content: A case study. In: 4th European Conference on Games Based Learning 2010, ECGBL 2010, (1)Katholieke Universiteit Leuven, 1 January 2010, pp. 40-46. Academic Conferences Limited.

Charlier N and De Fraine B (2013) Game-Based Learning as a Vehicle to Teach First Aid Content: A Randomized Experiment. Journal of School Health 83(7): 493-499.

Fortington L V, Bekker S, Morgan D, et al. (2017) 'It Doesn't Make Sense for Us Not to Have One'Understanding Reasons Why Community Sports Organizations Chose to Participate in a Funded Automated External Defibrillator Program. Clinical Journal Of Sport Medicine: Official Journal Of The Canadian Academy Of Sport Medicine. Australian Centre for Research into Injury in Sport and its Prevention, Federation University Australia, Ballarat, Australia.: Wolters Kluwer Health, Inc. DOI: 
Review Article

10.1097/JSM.0000000000000524.

Greenberg MR, Barr GC, Rupp VA, et al. (2012) Cardiopulmonary Resuscitation Prescription Program: A Pilot Randomized Comparator Trial. The Journal of Emergency Medicine 43(1). Elsevier: 166-171. DOI: 10.1016/J.JEMERMED.2011.05.078.

Huang Q, Hu C and Mao J (2016) Are Chinese Students Willing to Learn and Perform Bystander Cardiopulmonary Resuscitation? Journal of Emergency Medicine 51(6). School of Nursing, Tongji Medical College, Huazhong University of Science and Technology: Elsevier USA: 712-720. DOI: 10.1016/j.jemermed.2016.02.033.

International Federation of Red Cross Red Crescent Societies (2015) Law and first aid: Promoting and protecting life-saving action. Geneva, Switzerland: International Federation of Red Cross Red Crescent Socieities.

International Federation of Red Cross Red Crescent Societies (2016) International First Aid and Resuscitation Guidelines 2016. Geneva, Switzerland: International Federation of Red Cross Red Crescent Socieites. Available at: http://www.ifrc.org/Global/Publications/Health/First-Aid-2016Guidelines_EN.pdf.

Kliegel A, Scheinecker W, Sterz F, et al. (2000) The attitudes of cardiac arrest survivors and their family members towards CPR courses. Resuscitation 47(2): 147-154.

Moher D, Liberati A, Tetzlaff J, et al. (2009) Preferred Reporting Items for Systematic Reviews and MetaAnalyses: The PRISMA Statement. PLoS Medicine 6(7): e1000097. DOI:

10.1371/journal.pmed.1000097.

Pearn J, Dawson B, Leditschke F, et al. (1980) Who accepts first aid training? Australian Family Physician 9(9): 602-605.

Pellegrino JL, Oliver E, Orkin A, et al. (2017) A call for revolution in first aid education. International Journal of First Aid Education 1(1). DOI: 10.21038/ijfa.2017.0001. 
Review Article

Platz E, Scheatzle MD, Pepe PE, et al. (2000) Attitudes towards CPR training and performance in family members of patients with heart disease. Resuscitation 47(3): 273-280. DOI: S0300957200002458 [pii].

Prochaska JO and DiClemente CC (1983) Stages and processes of self-change of smoking: toward an integrative model of change. Journal of consulting and clinical psychology 51(3). UNITED STATES: American Psychological Assn: 390-395.

Roberts A, Nimegeer A, Farmer J, et al. (2014) The experience of community first responders in coproducing rural health care: in the liminal gap between citizen and professional. BioMed Central. DOI: $10.1186 / 1472-6963-14-460$.

Science Behind Our Programs | Science Advisory Council | Red Cross (n.d.). Available at: https://www.redcross.org/take-a-class/scientific-advisory-council (accessed 30 October 2019).

Singletary EM, Charlton NP, Epstein JL, et al. (2015) Part 15: First Aid: 2015 American Heart Association and American Red Cross Guidelines Update for First Aid. Circulation 132(18 suppl 2): S574-S589. DOI: $10.1161 / C I R .0000000000000269$.

Tricco AC, Lillie E, Zarin W, et al. (2018) PRISMA Extension for Scoping Reviews (PRISMA-ScR): Checklist and ExplanationThe PRISMA-SCR Statement. Annals of Internal Medicine 169(7): 467-473. DOI: 10.7326/M18-0850.

Yang MC, Fann CY and Huang CM (1998) Evaluation on the trainees of the first aid and CPR training course offered by Taipei Municipal Department of Health. Chinese Journal of Public Health 17(1). Yang, M.-C., School of Public Health, National Taiwan University, Taipei, Taiwan: 59-69.

Zacharatos A, Barling J and Iverson RD (2005) High-performance work systems and occupational safety. Journal of applied psychology VO - 90 (1). Washington, DC: American Psychological Association: 77. 